\title{
Two Heads Better Than One: Pattern Discovery in Time-Evolving Multi-aspect Data
}

\author{
Jimeng Sun ${ }^{1}$, Charalampos E. Tsourakakis ${ }^{2}$, Evan Hoke $^{4}$, Christos Faloutsos $^{2}$, \\ and Tina Eliassi-Rad ${ }^{3}$ \\ ${ }^{1}$ IBM T.J. Watson Research Center \\ ${ }^{2}$ Carnegie Mellon University \\ ${ }^{3}$ Lawrence Livermore National Laboratory \\ ${ }^{4}$ Apple Computer, Inc.
}

Data stream values are often associated with multiple aspects. For example, each value observed at a given time-stamp from environmental sensors may have an associated type (e.g., temperature, humidity, etc) as well as location. Time-stamp, type and location are the three aspects, which can be modeled using a tensor (high-order array). However, the time aspect is special, with a natural ordering, and with successive time-ticks having usually correlated values. Standard multiway analysis ignores this structure. To capture it, we propose 2 Heads Tensor Analysis (2-heads), which provides a qualitatively different treatment on time. Unlike most existing approaches that use a PCA-like summarization scheme for all aspects, 2-heads treats the time aspect carefully. 2-heads combines the power of classic multilinear analysis (PARAFAC [1, Tucker [5, DTA/STA [3, WTA [2] ) with wavelets, leading to a powerful mining tool. Furthermore, 2-heads has several other advantages as well: (a) it can be computed incrementally in a streaming fashion, (b) it has a provable error guarantee and, (c) it achieves significant compression ratio against competitors. Finally, we show experiments on real datasets, and we illustrate how 2-heads reveals interesting trends in the data.

This is an extended abstract of an article published in the Data Mining and Knowledge Discovery journal [4].

\section{References}

1. Harshman, R.: Foundations of the parafac procedure: model and conditions for an explanatory multi-mode factor analysis. UCLA working papers in phonetics 16 (1970)

2. Sun, J., Papadimitriou, S., Yu, P.: Window-based tensor analysis on highdimensional and multi-aspect streams. In: Proceedings of the International Conference on Data Mining (ICDM) (2006)

3. Sun, J., Tao, D., Faloutsos, C.: Beyond streams and graphs: Dynamic tensor analysis. In: KDD (2006)

4. Sun, J., Tsourakakis, C.E., Hoke, E., Faloutsos, C., Eliassi-Rad, T.: Two heads better than one: Pattern discovery in time-evolving multi-aspect data. Data Mining and Knowledge Discovery 17(1), 111-128 (August 2008)

5. Tucker, L.R.: Some mathematical notes on three-mode factor analysis. Psychometrika 31(3) (1966) 